\title{
Real heating-value based cost-accounting method with networking capabilities in natural gas distribution systems
}

\author{
G. Barta ${ }^{1}$ and T. Csubák Dr ${ }^{1}$ \\ ${ }^{1}$ Department of Control Engineering and Information Technology \\ Budapest University of Technology and Economics \\ Magyar Tudósok körútja 2, H-1117 Budapest (Hungary) \\ Phone number: +36 20328 9380, e-mail: barta@iit.bme.hu, csubi@iit.bme.hu
}

\begin{abstract}
Nowadays customer-side natural gas measurements are only focused on gas volume, while prices are increasing and the market becomes more open. Because of the efficient cost accounting, large customers get interested in measurements corrected by real calorific value. For determining the real energy flow, continuous measurement of gas consistency needed. But online gas chromatography is too expensive for even large consumers. However the information exists at provider-side databases and can be used for calorific value and energy flow computing at different customer-side locations. The aim of the research and development project was to give a cost-effective solution to these requirements. At the first we give a short theoretical overview of gas-flow measurements in cost-accounting and show the related standards. After that we define a realizable computing method, and deal with the structure and operation of the new measurement system.
\end{abstract}

\section{Key words}

data acquisition, cost accounting, natural gas measurement

\section{Introduction}

When dealing with gas flow measurements, especially in cost-accounting systems, we get strong accuracy criteria, which can only be met by real-time correction computations. For these we need not only physical parameters, but the actual consistency of the flowing media too.

If we assume that gas consistency does not change while travelling in pipes, the already realized provider-side consistency measurements can be used for calorific value and energy flow computing at cost accounting measurements on customer-side.

The new measurement system introduces a new communication and database structure in costaccounting. For realizing it, we need to create special hardware and software components, which are specified by the requirements described in the next sections.

\section{Background of corrected flow measurements}

The only fully standardized measuring principle for flow measurement is the differential pressure based method with orifice plates. The measurement can be easily reproduced because of its indirect principle. If the measuring system is constructed according to the standards, only the basic temperature and pressure sensors need to be calibrated, and the accuracy of the whole system can be determined theoretically.

The correlation between volume flow and the $\Delta p$ differential pressure measured between the two sides of the orifice plate (1) can be deduced from Bernoulli equation and the continuity equation. These are the two basic laws of flow theory.

$$
q_{V}=\alpha \cdot \varepsilon \cdot A_{0} \cdot \sqrt{\frac{2}{\rho} \cdot \Delta p}
$$

The flow coefficient $(\alpha)$ represents the geometry of the pipe and the orifice plate. In real cases it depends on the speed of the gas-flow, especially on the Reynolds number too. The expansion coefficient $(\varepsilon)$ is used to consider the change of speed, volume and density while the gas flows through the orifice. It depends also on the $\Delta p / p_{1}$ rate, where $p_{l}$ is the pressure before orifice. In technological control these coefficients can be defined as constants. But in cost-accounting systems they should be recalculated continuously, because of higher accuracy criteria.

Media density $(\rho)$ is also needed for calculating volume flow $\left(q_{V}\right)$. Direct measurement is rarely applied, but we have different ways to determine it. If density is known, mass flow $\left(q_{M}\right)$ can easily be calculated (2). 


$$
q_{M}=\alpha \cdot \varepsilon \cdot A_{0} \cdot \sqrt{2 \cdot \rho \cdot \Delta p}
$$

The structure of a differential pressure based gas flow measurement system is shown on Fig.1. Because of financial reasons, most parameters are usually calculated from a few directly measured parameters. Pressure and temperature measurements are reliable and based on simple principles. They provide high accuracy outputs, which are necessary for the cost-accounting purpose. However direct density measurements are very expensive, so it is usually calculated based on the consistency and the measured basic physical parameters.

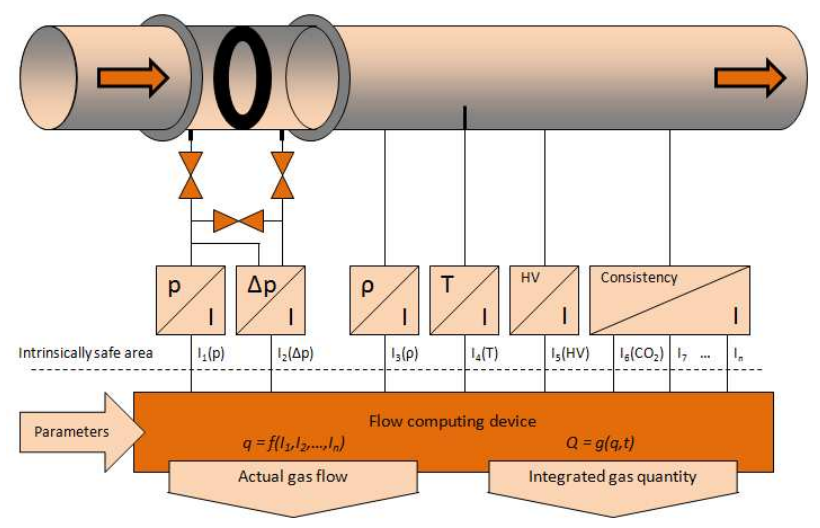

Fig. 1. Differential pressure based gas-flow measurement

Gas consistency is the next problem. Normally a chromatography system is used to get the actual proportion of contents, but measuring all of them is also uneconomical. That is why standard computing methods are used to get the needed data from a few measurements.

\section{Flow computing algorithm for embedded realization}

Fig. 2 shows the flow chart of a quick approximating gasflow computing algorithm [1] for embedded applications. The inputs are directly measured physical parameters (temperature and pressures) and measured consistency data, which need not cover all of the gascomponents. Processing physical measurements is done similar to other data acquisition systems. The measured temperature can be used to correct the pipe and orifice diameters for increasing accuracy.

The next section deals with calculating the proportion of missing gas-components, density and the calorific value of the gas, so now we assume that they are known values. As mentioned above, at cost-accounting purposes the expansion and flow coefficients should be recalculated continuously. For these calculations other parameters, like isentropic index and dynamic viscosity needed. They can be determined using empiric equations.

The most important step of the algorithm is the iteration cycle which determines the Reynolds number and the mass flow with the needed accuracy. For getting mass flow (2) the flow coefficient should be known. But it depends on Reynolds number, which changes by the mass flow. So at the beginning we estimate an initial value of the Reynolds number. With this, the flow coefficient and mass flow can be determined. This iteration cycle runs until the accuracy criteria (3) satisfies.

$$
\left|q_{M}(n)-q_{M}(n-1)\right|<0.0001 q_{M}(n)
$$

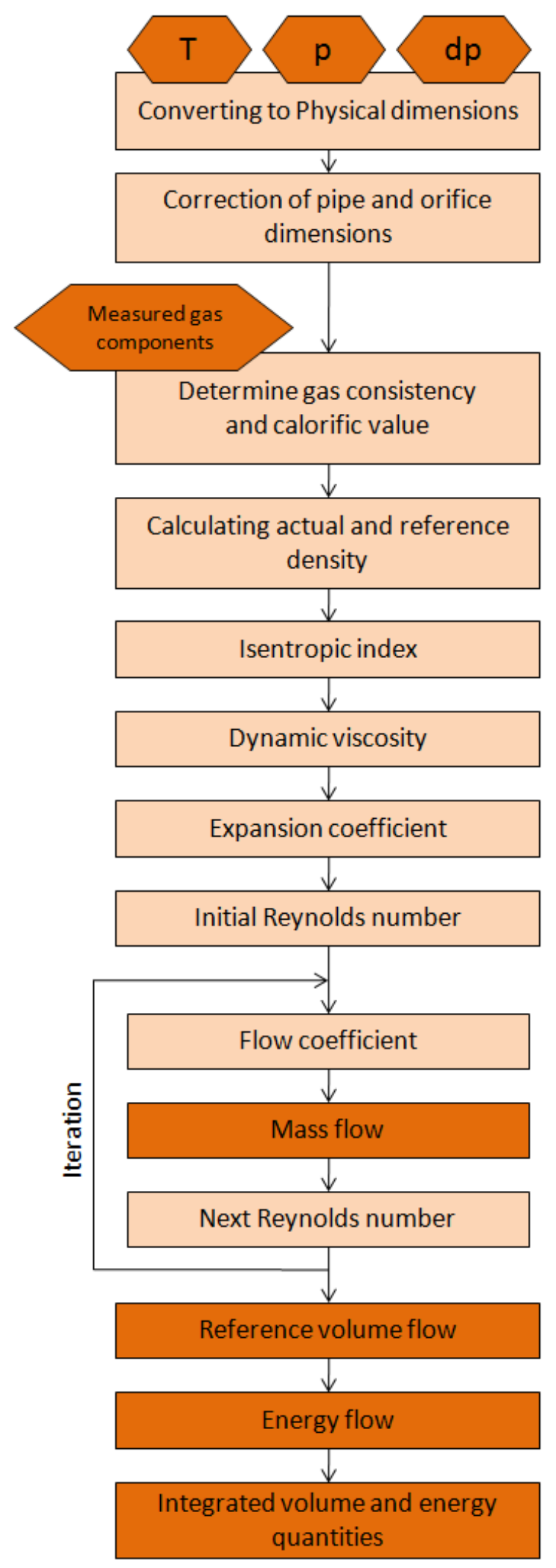

Fig. 2. Energy-flow computing algorithm 
When mass flow is known, volume flow can be calculated with the density. For cost-accounting the volume should be given at accepted reference temperature and pressure circumstances, so the reference density is needed instead of the operational one. Actual energy flow can also be calculated, because calorific value is known.

The actual mass, volume and energy flow are important parameters when we would like to know the actual state of the energy system, but for cost-accounting they should be integrated on a specified time interval (daily sum, monthly sum etc.) If it is done directly by the flow computing device, the measurement can easily be authenticated, which is essential need in the area of energy measurements.

\section{Computing with natural gas parameters}

For computing correction calculations a worldwide applied standard of the American Gas Association can be used [2] the short name of the standard is AGA-8. It standardizes the calculation of gas properties, enables to calculate the full consistency using a few measured parameters and gives standard accuracy ranges depending on the proportion of gas-components and physical conditions. For example if the temperature is between -51 and $+82{ }^{\circ} \mathrm{C}$, and the pressure is below 103 bar, the compressibility ratio can be calculated with the accuracy of $0.1 \%$ by using the defined methods.

If the measurements meet the accuracy criteria, and the computation (Fig.3.) is based on the standard equations, the system can meet the requirements of authentication, what is an essential need in cost-accounting.

First of all, if only partial consistency is measured, we should estimate the proportion of the unmeasured components. The standard gives different methods, which use some of the following input parameters:

- Relative density at $15.55^{\circ} \mathrm{C}$ and 1.015597 bar

- Average calorific value at $15.55^{\circ} \mathrm{C}$ and 1.015597 bar

- Mole fraction of $\mathrm{CO}_{2}$

- Mole fraction of nitrogen

- Mole fraction of methane

to determine the mole fractions of:

- Methane

- Ethane

- Propane

- Normal butane

- Isobutane

- Sum of pentanes

- Sum of hexanes and higher order hydrocarbons

- Sum of other components without $\mathrm{CO}_{2}$ and $\mathrm{N}_{2}$

The methods do not give any information about water and hydrogen-sulfide content of the natural gas.

After determining consistency, mole-density of the natural gas can be calculated by solving the stateequation (4).

$$
\begin{aligned}
p_{a b s} & =R \cdot T \cdot d+B \cdot R \cdot T \cdot d^{2}+C \cdot R \cdot T \cdot d^{3}+ \\
& +D \cdot R \cdot T \cdot d^{4}+E \cdot R \cdot T \cdot d^{6}+ \\
& +A_{1} \cdot R \cdot T \cdot d^{3}\left(1+A_{2} \cdot d^{2}\right) e^{-A_{2} d^{2}}
\end{aligned}
$$

Where $d$ is the mole-density, $T$ is the operation temperature, $p_{a b s}$ is the absolute operation pressure and $R$ is the gas constant. (Note that all the numerical values should be given in Anglo-Saxon units when using AGA-8 methods!)

$B, C, D, E, A 1$ and $A 2$ are the virial coefficients of the state equation. They denote the interaction of the gascomponents, and depend on the molecular weight, the energy, dimension and orientation parameters of the components and some empiric constants, given by the standard. To determine the virial coefficients, floating point multiplications and additions should be processed. These calculations need significant processor time in embedded systems, but can be solved without problems.

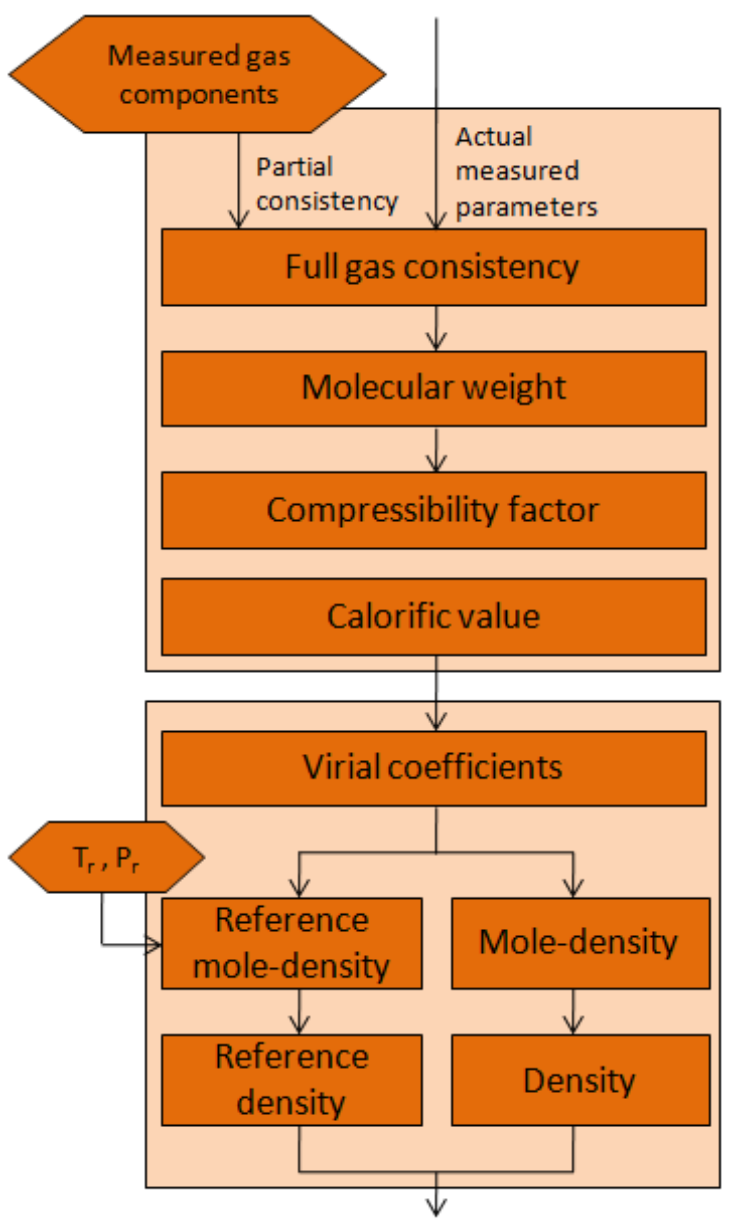

Fig. 3. Computing with natural gas parameters

A more considerable question is to solve the stateequation. It can be done only with a numerical method. If we denote the right side of the equation as $f(d)$ and define the functional $F(d)$ as

$$
F(d)=f(d)-p_{a b s}
$$


our task is to find the smallest root of $F(d)$. A bisection algorithm can be used. It is robust enough, but because of the embedded realization the needed processing time is an important question. The efficiency depends highly on the selected starting interval. If it is small enough, the searching will be fast, with guaranteed accuracy. For selecting the searching interval we specify the $d 0=0$, $d l=\Delta d, d 2=2 \Delta d, \ldots d k=k \Delta d$ points of the $d$ range, where

$$
\Delta d=0.1 \frac{p_{a b s}}{R \cdot T}
$$

The bisection searching can be started on the interval where the smallest $F(d)$ values were found, and with this condition the mole-density can be calculated with the needed accuracy in an admissible length of time.

For the cost-accounting we need the volume flow in reference circumstances, and for this calculation the reference density (7) should be determined.

$$
\rho_{r}=M \cdot d_{r}
$$

$d_{r}$ is the mole-density at the reference temperature and pressure, $\mathrm{M}$ is the molecular weight of the gas. It can be calculated using the measured or estimated consistency data. (8)

$$
M=\sum_{i} x_{i} \cdot M_{i}
$$

$x_{i}$ is the mole fraction, and $M_{i}$ is the molecular weight of the $\mathrm{i}^{\text {th }}$ component. Of course $\rho$ operational density can also be calculated instead of $\rho_{r}$ reference density. In this case the measured operational temperature and pressure should be substituted into the state equation instead of the reference ones.

One more property of the natural gas is needed to be able to calculate the energy flow. This is the actual calorific value (9).

$$
F=\frac{\sum_{i} x_{i} \cdot F_{i}}{Z_{b}}
$$

$x_{i}$ is the mole fraction, and $F_{i}$ is the calorific value of the $i^{\text {th }}$ component. $Z_{b}$ is the compressibility ratio of the natural gas in reference circumstances. It denotes the difference between the ideal gas and the real natural gas, and can be calculated from the consistency or from the density. If direct density or calorific-value measurements are applied and we have more possible ways to get the compressibility ratio - according to the standard - the consistency data should be used.

\section{Network structure of the measurement system}

The shown calculation assumes that at least partial consistency information are present at the flow computing device. Nowadays this condition is satisfied only at provider-side measurements, but some large customers are interested in real energy flow based costaccounting. Chromatography systems are too expensive, but if we assume that gas consistency does not change while travelling in pipes, the already realized providerside consistency measurements could be used. The structure of the novel, realizable system is shown on Fig.4.

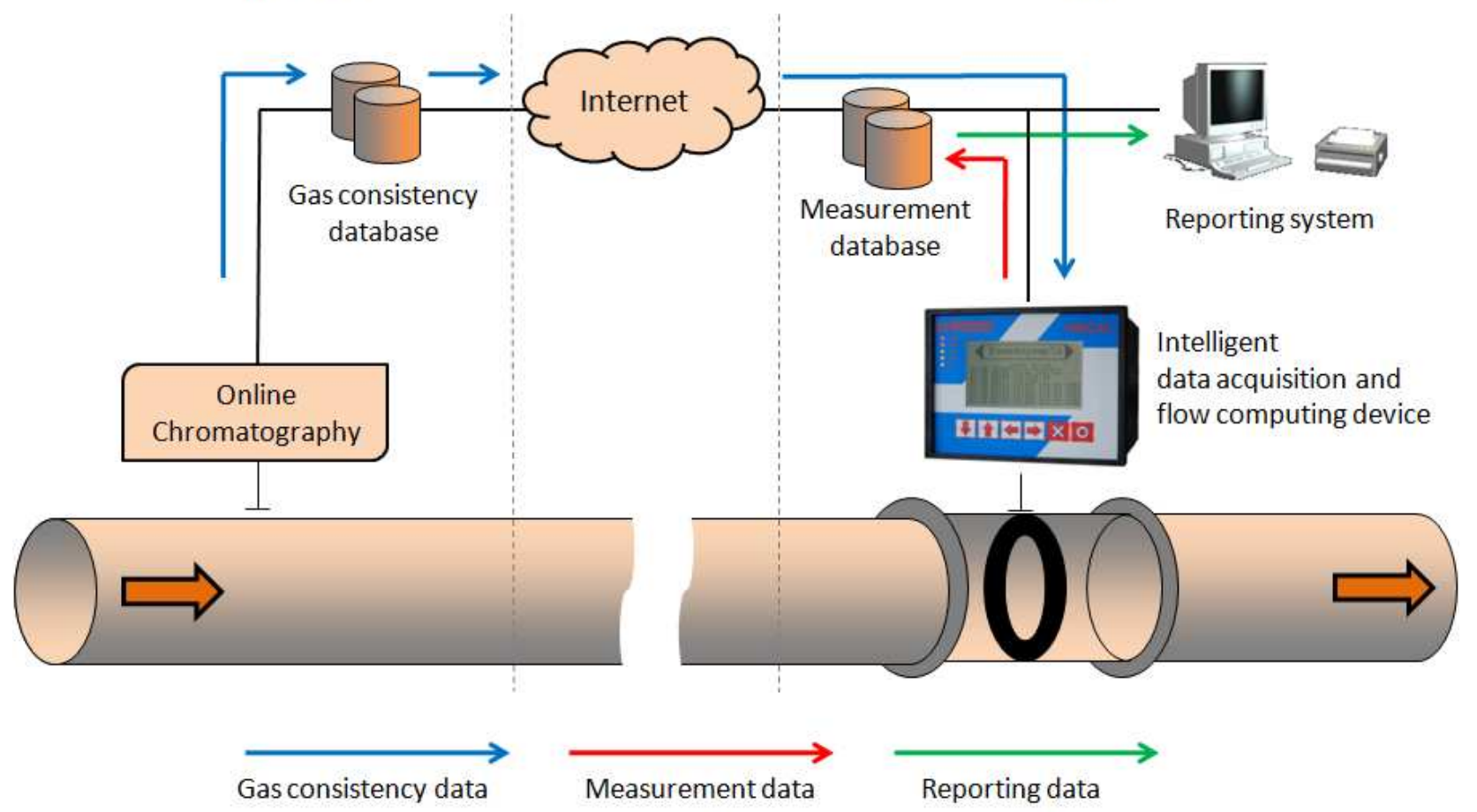

Fig. 4. Structure of the natural gas measurement system 
In this case the information should be transferred to different customer-side locations. The most cost-effective way is to use the Internet, but a secure transfer method should assure, that data arrives from the correct source and is not changed while its transfer. Ready to use solutions are used by IT providers for creating secure channels and transmitting the data, but communication errors should be handled automatically without interrupting the measurement system. Thus the received information should be stored locally in the data acquisition device. The embedded calculation algorithm uses the newest information for calculating calorific value and energy flow. The measured and calculated data are stored in the temporary storage of the device even for several days in the worst-case.

However in the normal way of operation measurement data is always uploaded and stored in the local measurement database. The connection between the device and the server should also ensure the security and integrity of the transmitted information. That is why special, non-public communication protocols can be proposed to realize over standard networking platforms, and there are also clever database-design aspects to use for protecting information against malicious interventions [3].

\section{The universal calculator for flow- measurements}

For realizing the new system an intelligent data acquisition device is required, which can be used for the differential pressure based flow measurement, and has computing capability enough for calculating with natural gas properties and solving the state equation.

It should be equipped with up-to-date communication interfaces for bidirectional data transfer, and because of legal regulations needs to agree with requirements of authenticated measurements. We analyzed both hardware and software aspects and developed the new UNICAL device. (Fig. 5.)

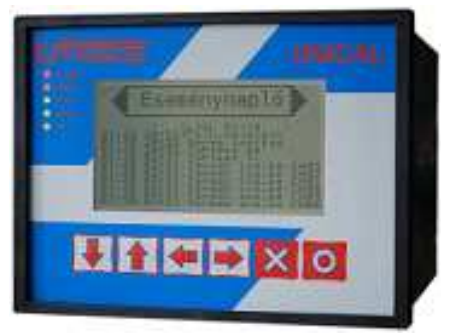

Fig. 5. UNICAL - The new intelligent data acquisition device for energy-flow measurements

The modular construction enables to use a wide range of input modules like analogue $0 . .20 \mathrm{~mA}$, RTD and pulse inputs. It can be integrated into the measurement system through its Ethernet connection, and can communicate with other appliances through an RS-485 serial line. If a wireless application needed, an additional GSM/GPRS modem can be used for connecting the device directly to the internet. UNICAL has industrial construction, it can be placed near to the process, typically into a control cabinet right after the IS barriers. It has a sophisticated user management system, because of data protection and for controlling user interactions, like parameter changes.

\section{A user-friendly output}

Nowadays a sophisticated and secure reporting subsystem is necessary for the clear, user-friendly operation. This enables to display actual and historical gas consumption data, system events, and of course creates the most important cost-accounting reports, which can be printed or even automatically transmitted to the enterprise management system. The applicable serverclient system has been successfully used in heat-flow measurement systems [4]. It runs on a common PC connected to the enterprise LAN, and can be customized as the user requires. For cost accounting purpose, a standalone system is easier to operate, but the stored data can be enabled to use in supervisory data acquisition systems or decision support applications.

\section{Conclusion}

The solution can capitalize the modern IT infrastructure to increase accuracy with the continuous automatic computing of real heating value and energy flow. The computing unit can easily be authenticated, because of the embedded calculating algorithm. Thus the system is an excellent solution for apply in modern cost accounting purposes. Now in the realization phase, the prototype of the data acquisition device has been developed, and the software components of the new system are under testing.

\section{Acknowledgement}

The R\&D project was sponsored by the National Office for Research and Technology.

\section{References}

[1] Dr. T. Csubák, "High-accuracy heat-flow measurement by using a quick approximate algorithm", Periodica Polytechnica Ser. El. Eng. Vol. 42, No. 2, Budapest, Hungary, 1998, pp. 233-250,

[2] American Gas Association: Transmission Measurement Committee Report No. 8 - Compressibility and supercompressibility for natural gas and other hydrocarbon gases, Arlington VA, USA, 1985

[3] Barta Gergely, Dr. Katona László, Dr. Csubák Tibor, "Validált tisztatéri monitoring rendszer és gyógyszeripari alkalmazása", in Proc. Factory Automation 2010, Kecskemét, Hungary, 2010, pp. 117-121

[4] Gergely Barta, Tibor Csubák, Dr, "Development of an intelligent data logging and computing device for heat flow measurement and integrate it into a supervisory acquisition system", in Proc. IYCE'09 2nd International Youth Conference on Energetics, Budapest, 2009, paper 33 